\title{
A service-based approach to nurse training in sexual and reproductive health care
}

\author{
Jill Shawe, ${ }^{1}$ Sarah Cox ${ }^{2}$ Nikki Penny, ${ }^{3}$ Alison White, ${ }^{4}$ \\ Christopher Wilkinson ${ }^{5}$
}

\begin{abstract}
${ }^{1}$ Senior Research Associate/ Specialist Nurse, Department of Sexual \& Reproductive Health, University College London, Institute for Women's Health, London, UK

${ }^{2}$ Specialist Nurse, Department of Sexual \& Reproductive Health, Central and North West London (CNWL) NHS Foundation Trust, Margaret Pyke Centre, London, UK

${ }^{3}$ Head of Nursing, CNWL NHS Foundation Trust, Margaret Pyke Centre, London, UK

${ }^{4}$ Clinical Services Manager CNWL NHS Foundation Trust, Margaret Pyke Centre, London, UK

${ }^{5}$ Lead Consultant CNWL NHS Foundation Trust, Margaret Pyke Centre, London, UK
\end{abstract}

\section{Correspondence to}

Dr Jill Shawe, Department of Sexual \& Reproductive Health, Institute for Women's Health, London, UK;

j.shawe@ucl.ac.uk

Received 14 September 2012 Revised 21 December 2012 Accepted 8 January 2013

5 February 2013 University College London,

Published Online First

\section{ABSTRACT}

Aims To develop and pilot a reproducible curriculum for nurses with limited or no sexual and reproductive health care $(\mathrm{SRH})$ experience to become competent dual-trained practitioners. The model used supernumerary training posts and clinical training and assessment adapted from that used to train doctors for the Diploma of the Faculty of Sexual \& Reproductive Healthcare in the UK.

Background There is currently no standard SRH nurse training in the UK. Nurses undertake various university-based qualifications supported by clinical sessions, and employers can be reluctant to sponsor these because of the cost and the time required.

Methods Two nurses were employed on a 6-month programme consisting of five parts: (1) the Faculty of Sexual \& Reproductive Healthcare (FSRH) e-learning programme, in conjunction with e-Learning for Healthcare;

(2) the FSRH 'Course of 5'; (3) structured clinical training; (4) visits to other relevant services; and

(5) clinical supervision and mentoring.

Assessment was by a portfolio of evidence; signed competences; reflections from clinical sessions; and outcomes of visits to services. The project was evaluated by means of questionnaires and interviews with trainees and staff.

Conclusions The project demonstrated that by using a model similar to that used for basic SRH medical training it is possible to train nurses in core holistic SRH care within the workplace. Many SRH services are reporting severe difficulties in the recruitment of nurses trained in $\mathrm{SRH}$, and this practice-based training would allow services and providers to invest in training to build up the workforce they require.

\section{INTRODUCTION}

Penny N, et al. J Fam Plann Reprod Health Care

2013;39:285-288.
Nurses working in the field of sexual and reproductive health care $(\mathrm{SRH})$ have

\section{Key message points}

- In order to meet current workforce demands, new models are required for training nurses in sexual and reproductive health care (SRH).

- This pilot used supernumerary training posts and a curriculum based on the Diploma of the Faculty of Sexual \& Reproductive Healthcare to demonstrate that nurses with limited or no SRH experience can become competent dual-trained practitioners within 6 months.

traditionally undertaken training via part-time structured sexual health courses. These originally had a standardised national curriculum and were validated by the National Boards for Nursing, Midwifery and Health Visiting until 2002. Once the boards were disbanded, universities met the need for training by individually developing programmes that vary in academic level, content, length and cost. ${ }^{1}$ Most courses focus on either 'contraception' or 'sexually transmitted infections' rather than reflecting the current service model of integrated sexual health care. ${ }^{2}$ It can take up to a year to complete both courses and employers are reluctant to release nurses to attend extensive classroom-based training. The amount of clinical experience offered within these courses varies and may not be sufficient to ensure that nurses are competent in the clinical skills required for their current extended role. ${ }^{1}$

This project aimed to move away from the traditional classroom and sessional practice-based model. A curriculum was developed and piloted for nurses with limited or no SRH skills, employed in supernumerary 'training posts' with support to gain the required skills to work independently to become competent dual-trained practitioners in SRH. 
Many SRH services are reporting severe difficulties in the recruitment of nurses trained in $\mathrm{SRH}^{2}$ and this practice-based training would allow services and providers to invest in training to build up the workforce they require.

\section{METHODS}

Two nurses with limited SRH experience were appointed into 6-month full-time Band 5 training posts at the Margaret Pyke and Mortimer Market Centres in London, UK [Central \& North West London (CNWL) NHS Foundation Trust]. The centres are Level $3 \mathrm{SRH}$ and genitourinary medicine (GUM) services with some integrated services.

\section{Curriculum}

The curriculum was based on the model used to train doctors for the Diploma of the Faculty of Sexual \& Reproductive Healthcare (DFSRH) in the UK. ${ }^{3}$ This covers routine contraception, early pregnancy assessment and referral, and sexually transmitted infection (STI) diagnosis and management in men and women. The programme aimed to equip nurses to deliver holistic modified Level $1 \mathrm{SRH}$ care (Box 1), including assessment and provision of some long-acting reversible contraception (LARC) methods. Although the curriculum had similarities to the nurse training described by Mehigan and Burnett ${ }^{4}$ in Berkshire, UK, it differed in the method of assessment and the fact that the nurses were employed full-time as supernumerary trainees within the SRH service.

The training programme consisted of five parts: (1) the FSRH e-learning programme (e-SRH), in conjunction with e-Learning for Healthcare; (2) the FSRH 'Course of 5'; (3) structured clinical training; (4) visits to other relevant services; and (5) clinical supervision and mentoring.

\section{Assessment}

Each nurse was allocated two nurse specialists as clinical assessors, one for GUM and one for contraception. The DFSRH e-portfolio is not available to

\section{Box 1 Basic sexual and reproductive health care}

- Sexual history and risk assessment

- The law and sexual health

- Recognising psychosexual issues

- Contraception: assessment and provision of most reversible methods, and assessment and referral for natural family planning, implants and intrauterine methods

- Asymptomatic and symptomatic sexually transmitted infections (male and female)

- HIV testing and counselling

- Hepatitis B immunisation

- Young people

- Cervical cytology screening and referral

- Pregnancy testing and referral nurses and assessment was by a portfolio of evidence demonstrating the following achievements: successful completion of the e-learning and Course of 5; demonstration of competence using Assessment of Clinical Practice (ACP) tools signed off within a logbook developed by the programme management team; reflections from clinical sessions; and outcomes of visits to services.

\section{Evaluation}

A nurse specialist acted as a mentor to the trainees for the theoretical part of the programme and as an external reviewer for the progress and evaluation of the project. A steering group of nurses and a doctor oversaw the project. The project was formally evaluated by means of questionnaires and interviews with both trainees and the staff involved at three points in the project. In addition the trainees completed a questionnaire at the end of the programme and had a final 'sign off' interview.

\section{FINDINGS}

The project was evaluated highly by both trainees and staff and the key themes are outlined below.

\section{e-learning}

Neither trainee had used e-learning before and both had been given protected time to work through each module.

\begin{abstract}
"The best thing was everything related well - doing the e-learning in the morning and then putting it into practice in the afternoon!"

"I was given dedicated study time to complete the elearning programme and I would spend approximately half of each day on theory and half in practice for the first few months of the training. This worked really well."
\end{abstract}

\section{Course of 5}

The actual experience of the Course of 5 was very positive but there was some anxiety because the trainees attended a multidisciplinary course with general practitioners (GPs) and other doctors.

"I felt particularly nervous about the Course of 5, mainly because I was aware of the fact that I would be doing the course with GPs."

The trainees felt that they were well supported and that the course was overall very beneficial.

"I learnt a lot from the other candidates as well as the facilitator, and feel that I had sufficient experience enabling me to participate fully."

"The Course of 5 made me realise how much information I had actually retained from the e-learning, and made me more confident, which I wasn't expecting." 


\section{External visits}

The trainees found the external visits very useful but would have found it useful to have specific learning objectives.

"There were no clear objectives set on the visits. I assumed the point was to gain information into the services available for patients, which would make it more effective for referring patients to these services and letting them know the procedures, etc."

\section{End of programme evaluation}

The trainees had a good overall experience of the programme and were complementary about the supportive environment for training from mentors, assessors and the clinical staff.

"I have felt well supported by all staff I have had the opportunity to work with. They have all been supportive and encouraging in my learning and development."

"Although when I first began to take consultations on my own I felt anxious, I was extremely well supported throughout."

Both trainees would recommend the programme to other nurses wishing to work in the field of SRH.

"This is a great opportunity for nurses who wish to enter the field of sexual and reproductive health. Although the programme requires lots of selfmotivation and organisation, and some of the theory was intense, I have really enjoyed the programme overall and would recommend it to any nurse who wants to enter the field."

\section{Comparison with traditional university sexual health courses}

In comparison to students taking a traditional university sexual health or contraception course, staff felt that this programme was advantageous in having the integration of theory and practice and more time spent in practice overall.

"I think this course was way ahead because I think somebody doing the traditional course, they still have quite a basic knowledge and they haven't had time to consolidate that knowledge. So, I think by doing it altogether it is by far superior."

One member of staff felt that the programme produced trainees who were competent in 6 months in comparison to employing a new member of staff who had undertaken a sexual health course.

"So my feeling is that, although they have the [university] course if they then come in as a new staff member, they would still need to have a certain period of time to consolidate so it would just take longer. I think this 6-month programme has shown that actually within the 6 months they can be a competent dualtrained practitioner by the end of the course."

\section{Accreditation}

The trainees had concerns that other employers might not recognise the training; however, the National Health Service (NHS) Trust managers who employ nurses felt that the fully completed logbook and portfolio would serve to demonstrate competence.

\section{DISCUSSION}

Many SRH services are reporting severe difficulties in the recruitment of nurses who are competent in the skills required to be able to work independently within new models of integrated care. This project was set up with the aim of training nurses in-house with limited or no experience in SRH to become competent practitioners offering both SRH and GUM integrated care within a 6-month timeframe.

The trainees had been attracted to the programme as it was a supported way to develop and gain the skills required to work within an integrated sexual health service. Both trainees had some limited experience of sexual health nursing in the past - one in HIV nursing and the other within a GUM clinic - which may have positively influenced the development of their skills and their ability to work within a clinic setting.

\section{e-learning}

Both trainees started the programme by undertaking the e-learning modules and integrating these with observed clinical practice. They found it best to spend half a day at a time at the computer and then consolidate their learning within a clinical practice environment for the remainder of the day. Neither trainee had used e-learning before and both completed the elearning within 2 months as had been predicted at the onset of the programme The staff found that the knowledge base of the trainees after they had completed the e-learning was an excellent foundation for building clinical skills, and the trainees were able to advise patients and provide up-to-date information within their clinical sessions.

\section{Course of 5}

This was the first time that nurses had attended the Course of 5 locally, and all those involved in the process thought it had been a very positive experience. There was some initial concern on the part of the medical facilitators that the knowledge levels of the trainees might be inadequate in comparison to medical staff attending the course, and this was reflected in the nurses' anxiety prior to attending the programme. This concern turned out to be unfounded, and in some areas the nurses were found to have better knowledge than the medical trainees.

Future courses may require tailoring to the needs of the nurses as they are not required to undertake certain skills such as bimanual examinations at this stage. The nurses felt that the Course of 5 had 
enhanced their confidence and that it was useful to have a formal assessment which helped identify areas for further development.

\section{External visits}

The trainees arranged their own visits, and although these had been very valuable there had been some difficulty in contacting some services. In the future it may be better if the programme team establishes more formal arrangements with relevant services.

\section{Achieving competency}

The trainees achieved the necessary competences after 4-5 months and then were supervised in the consolidation of their practice. Both trainees already had some limited experience in sexual health nursing and most of the staff agreed that a 6-month timeframe would be reasonable for someone without any sexual health background to achieve integrated competences. As the trainees' independence increased so did their delivery of routine service clinical care, and subsequent cost-benefit analysis demonstrated that the 'trainee' scheme gave a greater return over the 6-month period, with nurses working independently by Month 4 compared to after Month 6 for a traditionally dual-trained student.

\section{Accreditation}

Currently the programme is not accredited by a higher education institution (HEI) and the trainees had concerns that employers might not recognise the training. Managers in the CNWL NHS Foundation Trust who employ SRH nurses felt that they would take the logbook and portfolio as an appropriate demonstration of competence to practise; and as was raised in the evaluation of the Berkshire model, ${ }^{4}$ accreditation does not guarantee uniform clinical competence. Trainees would also have liked credits towards a degree or postgraduate qualification. HEIs are becoming more flexible towards recruitment into their programmes and accreditation of prior experiential learning is possible. Alternatively, accreditation for the Margaret Pyke Programme through collaboration and partnership with an HEI could be sought.
Funding and reproducibility

This pilot was funded by a grant; however, due to the successful outcome and positive recommendations from the steering group the Trust have indicated their support to continue the programme to build an effective workforce. The authors believe that this model of training should be deliverable in most areas where SRH and GUM services are already working together to deliver DFSRH training.

\section{CONCLUSIONS}

The project has proved to be a success and that this approach is a valid model for training future SRH nurses. Both nurses completed the programme within the 6-month timeframe and impressed colleagues with their competence and confidence as valuable members of the SRH team. Both individuals have subsequently been employed within the service as integrated SRH nurses. The development and funding of further training posts is a priority; and in order to confirm that dual training can absolutely be achieved within a 6-month timeframe, nurses without any sexual health experience should be recruited for the next programme.

Funding This project was funded by the London Sexual Health Programme by a grant awarded as a result of a competitive bidding process.

Competing interests None.

Provenance and peer review Not commissioned; externally peer reviewed.

\section{REFERENCES}

1 Mehigan S, Moore W, Hayes L. Nurse training in sexual and reproductive health. J Fam Plann Reprod Health Care 2010;36:5-6.

2 Estacourt C, Tyler C, Nelson M, et al. Challenges facing nurse-led STI care and sexual and reproductive health: education, education, education! Sex Transm Infect 2011;87:453.

3 Faculty of Sexual \& Reproductive Healthcare. Diploma of the Faculty of Sexual \& Reproductive Healthcare (DFSRH). 2012. http://www.fsrh.org/ [accessed 20 Dec 2012].

4 Mehigan S, Burnett J. An innovative training for nurses in sexual and reproductive health. J Fam Plann Reprod Health Care 2012;38:194-195. 